\title{
Breakage of flawed particles by peridynamic simulations
}

\author{
Nicolas Blanc ${ }^{1} \cdot$ Xavier Frank $^{1} \cdot$ Farhang Radjai $^{2} \cdot$ Claire Mayer-Laigle $^{1} \cdot$ Jean-Yves Delenne ${ }^{1}(\mathbb{C}$
}

\begin{abstract}
In this paper, we use a 2D bond-based peridynamic model to investigate the strength of disk-shaped particles including pre-cracks. We use a diametral (or Brazilian) test to break the particles. For the flawless particles, we find that the stress distribution compares well with an analytical model accounting for the size of the contact zone, and the particle stiffness tends linearly to a well-defined value for increasingly resolved meshing. We then introduce cracks, which are numerically defined by reducing the Young modulus of the bonds crossing linear segments. We consider in detail the effect of a single vertical crack on the yield stress as a function of its position. We also consider a randomly distributed population of cracks with sizes generated from a Gaussian size distribution. For a parametric study with a hundred particles, we found a probability of failure that is well fit by a Weibull law. Finally, using an image analysis algorithm, we investigate the statistics of cracks and the resulting fragments.
\end{abstract}

Keywords Particle breakage $\cdot$ Flaws $\cdot$ Peridynamics $\cdot$ Crack patterns

\section{Introduction}

In chemical engineering, the powder particles can be the result of various down-sizing or up-sizing processes such as grinding, micronization, erosion, agglomeration, atomization, and RESS (Rapid Expansion of Supercritical Solutions). These particles may present a wide range of bulk or interface properties, and they are most often broadly distributed with various shapes.

An important issue is to evaluate the strength of these particles depending on the material and mechanical loading. This strength plays a key role in many practical problems such as the minimization of energy to achieve a given size reduction or the reduction in fine particles resulting from powder processing. In the same vein, it is well known that the strength highly depends on the internal microstructure of the particles. For example, the strength of compacted powders depends on the porosity [1,2] or in cohesive granular aggregates it also depends on the amount of gluing matrix

We thank BPI France for their financial support.

Jean-Yves Delenne

jean-yves.delenne@inrae.fr

1 IATE, Univ Montpellier, INRAE, Institut Agro, Montpellier, France

2 LMGC, Univ Montpellier, CNRS, Montpellier, France
[3]. Although homogenization methods have been used to account for the effect of heterogeneities [4], they usually fail in predicting the influence of singularities such as cracks or bare contacts on the onset and propagation of cracks.

For practical reasons, the diametral compression, which consists in crushing a grain in compression between two plates, is frequently used for the evaluation of the strength of particles $[5,6]$. This test gives an indirect but easy-tohandle measurement of the tensile strength. Both disk (or cylindrical)- [7] and sphere-shaped [8] particles were tested but also non-spherical grains [9]. This test has been successfully employed in many practical issues in civil engineering, pharmaceutical tableting, strength of ore granules, as well as in more fundamental studies of the influence of the microstructure on fracture and fragmentation $[10,11]$.

Numerical simulations based on the Discrete Element Method (DEM) have been used for the investigation of particle crushing by modeling each grain as a cohesive agglomerate of smaller (primary) particles [12-15]. Although these approaches fundamentally consider the material as a discrete medium with potential pre-existing fragments, the evolution of cracks and complex geometries of fragments in 2D [16-18] and 3D are reproduced with rather good accuracy [19-22].

However, in DEM-type approaches, such as cohesive DEM [16] or bonded-cell [17,23] methods, the crack path 
is strongly guided by the characteristic size of the DEM elements or by an underlying tessellation of the particles at the microscale. Due to the non-local character of peridynamics the crack propagation is less affected by the local ordering induced by the angular distribution of the bond orientations [24-26]. In this paper, we use such a method applied to particles that can break into fragments of arbitrary sizes and shapes (up to the discretization limit) in the presence of microcracks. Peridynamics has been successfully used for modeling the fracture of materials such as concrete [27], nickel nanowires [28], and wood [29]. It was introduced by Silling [30] as an alternative method to continuum mechanics based on integro-differential equations instead of differential equations. This framework allows one to include discontinuities such as pores, cracks or stiffness, and damage gradients without involving any particular case-sensitive processing for each problem [30]. Although it can be CPU time consuming, peridynamics exhibits a weaker dependence with respect to the discretization grid contrary to other lattice methods that consider only the nearest neighbors such as the Fuse Models [24], the Lattice Element Method (LEM) [25], or peridynamics approaches computed for $h=1 \delta x$ [26].

In this paper, we use a bond-based implementation of peridynamics to investigate the crack patterns of 2D disk-shaped particles embedding flaws. The population of flaws is randomly distributed with a controlled density. The particles are loaded in compression between two horizontal plates. We are interested in the probability distribution of yield stresses and fragments for a large number of particles.

\section{Bond-based peridynamics}

In our model, we consider that the domain is discretized using material points located on a $N_{x} \times N_{y}$ grid with a spatial resolution $\delta x$ (Fig. 1). In the following, we assume $N_{y}=N_{x}$. A volume $V_{i}$ and a mass $m_{i}=\rho V_{i}$ (where $\rho$ is the density of the material) are associated with each point $i$ of position $\boldsymbol{x}_{i}$. The point $i$ interacts with its surrounding nodes $j$ of position $\boldsymbol{x}_{j}$ within a distance called the horizon $h$. Hence, this horizon contains all nodes $j$ belonging to the set

$\mathcal{H}_{i}=\left\{j,\left\|\boldsymbol{x}_{j}-\boldsymbol{x}_{i}\right\|<h\right\}$

The vector $\boldsymbol{\xi}_{i j}=\boldsymbol{x}_{j}-\boldsymbol{x}_{i}$ is the relative position between the two nodes defined at the initial state, and we denote by $\boldsymbol{\eta}_{i j}$ their relative displacement after deformation.

In bond-based peridynamics, the 1D elements called "bonds" connecting the material points only transmit independent pair-wise forces. We assume in this paper a linear relationship

$f=c s n$ where $s=(\|\boldsymbol{\xi}+\boldsymbol{\eta}\|-\|\boldsymbol{\xi}\|) /\|\boldsymbol{\xi}\|$ is the bond elongation, $\boldsymbol{n}=(\boldsymbol{\xi}+\boldsymbol{\eta}) /\|\boldsymbol{\xi}+\boldsymbol{\eta}\|$ an orientated unitary vector and $c$ the so-called micromodulus. For elastic materials described in plane stress, $c=6 E /\left(\pi h^{3}(1-v)\right)$ [31], where $E$ is the Young modulus and $v$ is the Poisson coefficient. Note, however, that for theoretical reasons $[30,32]$ and similar to other lattice approaches $[25,33] v$ is fixed in the bond-based model (value is $1 / 3$ in $2 \mathrm{D}$ and $1 / 4$ in $3 \mathrm{D}$ ). A more in-deep discussion on the way Poisson's ratio is related to the connectivity of the lattice bonds can be found in [34]. It is also possible to overcome this limitation using enriched models $[35,36]$. However, as $1 / 3$ is a reasonable value for many materials such as many polymers (PPMS 0.34, polystyrene 0.35, PMMA $0.36 \ldots$ ) [37], metals (copper 0.33, aluminum 0.32 ...) [38], or geopolymers [39], in this study, we focus on the effect of inhomogeneities which play a dominant role on the failure mechanisms.

The material can be viewed as a mass-spring system where material points interact through harmonic bonds. The time evolution of such systems can be computed using the velocity-Verlet algorithm [40] with a time step $\delta t$. To dissipate the elastic waves, a damping force $\boldsymbol{F}_{i}^{d}=\eta \dot{\boldsymbol{x}}_{i}$ is applied on each node, where $\dot{\boldsymbol{x}}_{i}$ is the velocity of the material point $i$ and $\eta$ a viscosity coefficient. This latter should be chosen below the minimal critical damping value $\eta_{\mathrm{c}}$. In the same vein as other dynamic models based on mass-spring systems, the critical damping $\eta_{\mathrm{c}}$ is the value of the viscosity above which the bond between two neighbors is overdamped. The value of the damping was chosen to damp the elastic waves during the quasi-static loading phase without influencing the crack propagation at breakage. Note, however, that the yield stress, investigated in details in this study, is almost not influenced by the damping which was fixed to $\eta=\eta_{\mathrm{c}} / 10$ for all simulations.

The occurrence of fracture in the material can be taken into account by introducing a critical elongation $s_{0}$ above which the bonds fail. For plain stress [31]

$s_{0}=\sqrt{4 \pi G /(9 E h)}$

where $G$ is the macroscopic fracture energy of the material. Due to the non-local description of peridynamics, it is usually convenient to consider a damage parameter. Assuming that the cell volumes do not vary significantly, this damage can be evaluated at each node as the ratio between the number of broken bonds and the initial number of bonds connected to the node.

A horizon $h=3 \delta x$ is used in the following, corresponding to 28 connections for bulk nodes (which are at a distance greater than $3 \delta x$ from the boundaries). According to several authors who investigated the sensitivity of macroscopic elastic properties to the horizon size in discrete peridynamics approaches [41,42], this value is a good compromise 
Fig. 1 A disk particle discretized by a rectilinear grid of nodes (512 nodes per diameter) with two successive zoom-ins on the boundary of the disk and a node with all its bonds within a peridynamic horizon $h=3 \delta_{x}$

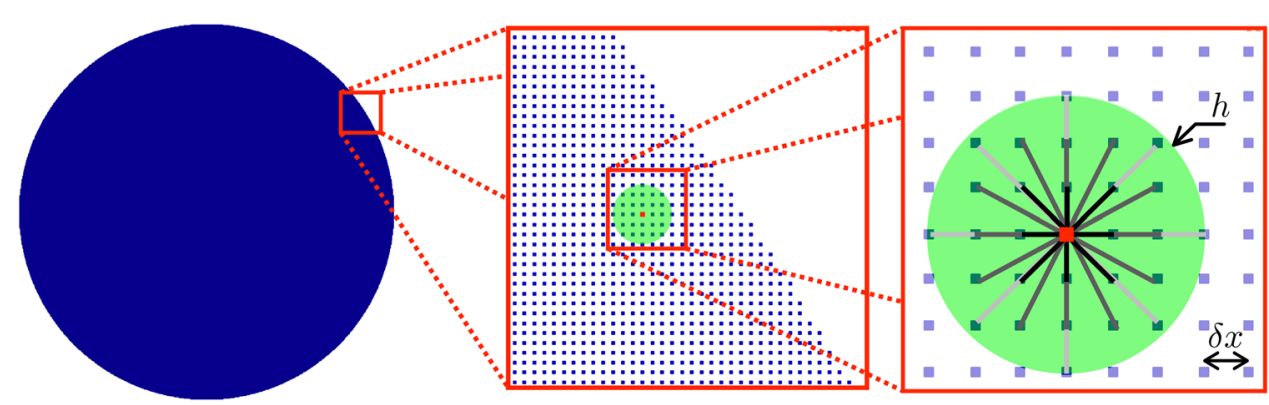

between the accuracy of the solution (in the sense of continuum mechanics prediction and real experimental values), which increases with the size of the horizon, and the computational efficiency, which declines as the size of the horizon increases. The material parameters $E$ and $G$ are kept constant for all simulations mentioned in our study.

\section{Diametral compression test}

The diametral compression test is frequently used for the experimental evaluation of the strength of particles. For different practical problems, it has been simulated using many numerical approaches, for example, the Finite Element Method [43], the Discrete Element Method [44,45], Contact Dynamics [46], peridynamics [47,48], Material Point Method [49], Phase Field simulations [50]...

In this study, we performed diametral compression tests of disk-shaped particles of diameter $D=2 R$ between two plates. These particles are discretized on a rectilinear grid of $512 \times 512$ nodes using a simple cutoff procedure. They are then subjected to a diametral compression between two plates (Fig. 2). The plates, initially separated by a distance $\ell_{0}=D$, are moved inward. The macroscopic vertical strain is defined by

$\varepsilon=-\frac{\ell-\ell_{0}}{\ell_{0}}$

where $\ell$ is the distance between the two plates. The top plateau is connected via bonds of a given stiffness $k_{\mathrm{w}}$ (wall stiffness), which is 100 times that of particle bonds, to the nodes belonging to the periphery of the particle. The plateau is moved downward at constant speed. With a linear elastic behavior, a normal force $k_{\mathrm{w}}\left(\delta-\delta_{0}\right)$ is added to each node at a distance $\delta$ from the wall, where $\delta_{0}$ is the reference length of the bond. For each wall, the total resulting vertical force $F$ exerted on the particle is equal to the total force acting on the wall, obtained by summing all the contributions of the boundary nodes. For the particle-plate friction, we considered a regularized Coulomb friction law [51] with a coefficient of friction $\mu=0.5$.

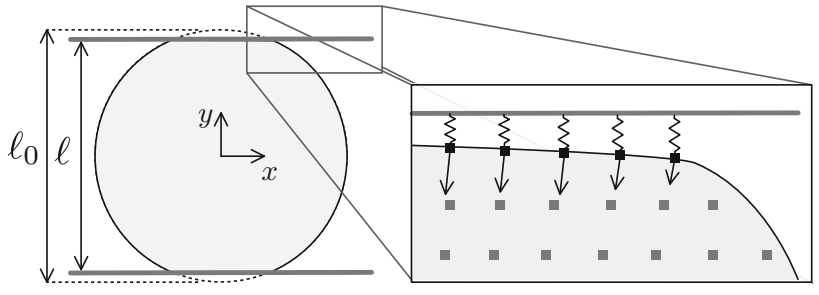

Fig. 2 Diametral compression test on a particle between two rigid plates. Schematic representation of the contact area

\subsection{Stress-strain curve}

The applied strain rate should be a compromise between the simulation time and the propagation time of the elastic waves in the lattice mass-spring system. In the following, it is fixed to a value equal to $0.07 \%$ of the lattice speed to reduce the inertial effects. Figure 3 shows a typical stress-strain curve where the average stress $\sigma=F / D$ is normalized by the theoretical stress at failure

$\sigma_{t}^{Y}=\frac{F^{Y}}{\pi R}$

for a rupture force equal to $F^{Y}$ [52]. The curve is nonlinear, the change in slope being due to the rasterization of the particle. This effect tends to vanish at increasingly higher resolution. We see that rupture occurs for $\sigma^{Y} \simeq 1.4 \sigma_{t}^{Y}$. This is because we have set here $\sigma=F /(2 R)$, and failure occurs for $F=F^{Y}$. Hence, according to Eq. (5), we have $\sigma^{Y} / \sigma_{t}^{Y}=\pi / 2$ at failure. After breakage, the plates are stopped to avoid additional breakage of fragments. We define the damage rate $\lambda(\varepsilon)$ as the ratio of the number of broken bonds for a deformation of $1 \%$ to the initial number of bonds. An example of cumulative damage $100 \int \lambda(\varepsilon) \mathrm{d} \varepsilon$ is shown in Fig. 3 as a function of cumulative deformation. The damage rate begins to increase rapidly at $\varepsilon \simeq 1.9 \%$ as the stress is approaching its peak value. Then, it vanishes rapidly with stress drop. To allow a full crack pattern development, the simulation was allowed to run until the damage rate is below $\lambda=0.002$. 


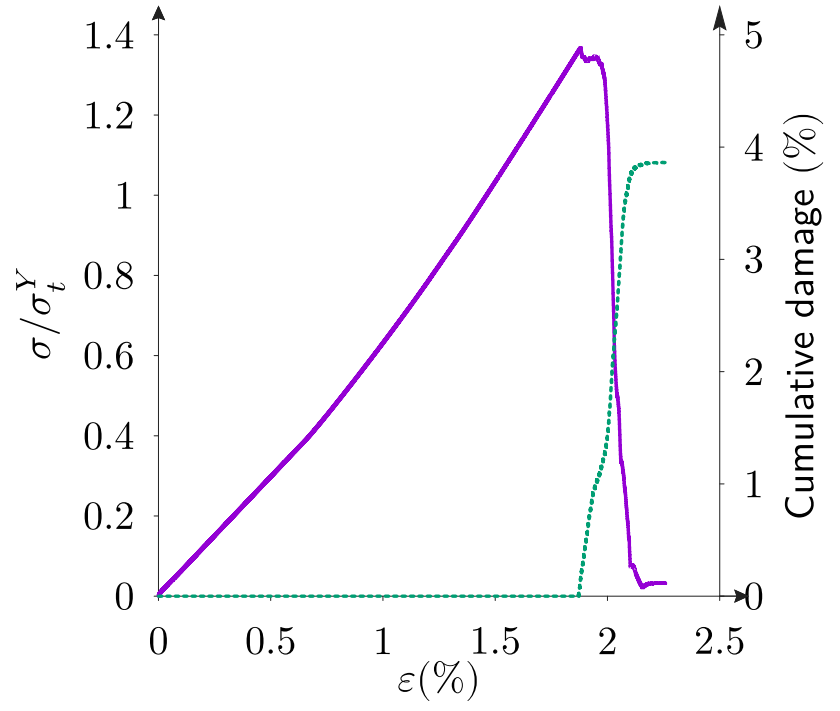

Fig. 3 Vertical stress (purple curve, solid line) and cumulative damage (green curve, dashed line) as a function of cumulative vertical strain for a particle meshed with $N_{x}=512$. The stress is normalized by the theoretical failure stress $\sigma_{t}^{Y}$

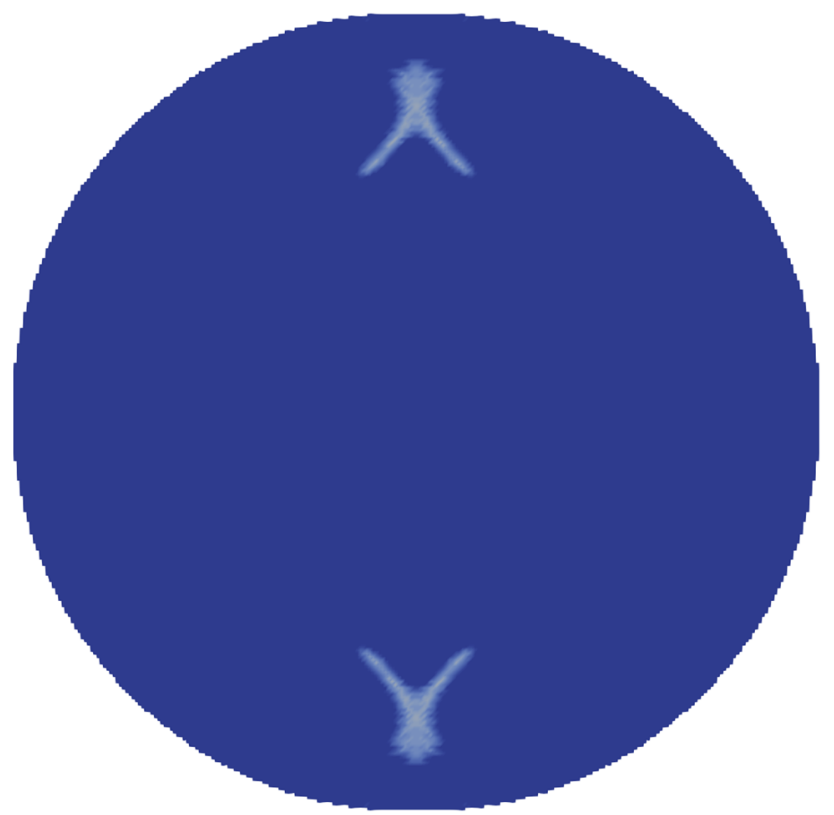

Fig. 4 Damage zone (in light blue) showing the onset of fracture

Interestingly, as observed in Fig. 4, the onset of fracture is not located at the maximum tensile stress but at $y / D= \pm 0.375$ corresponding to points where the Tresca equivalent stress $\max \left(\sigma_{\mathrm{I}}-\sigma_{\mathrm{II}}\right)$, where $\sigma_{\mathrm{I}}$ and $\sigma_{\mathrm{II}}$ are the principal stresses, is maximum. The slip plane is nearly at $45^{\circ}$ to the compression axis, as expected for an amorphous material. This indicates that the particle does not break in pure tension but in shear, as it was also observed in FEM tests [53]. The damaged zone is symmetrical, which is consistent with the fact that no defects are present in the particle. We note also a bifurcation of the crack path showing a dynamic propagation after the onset of failure. Indeed, prior to fracture the stress concentration zone is large and the elastic energy stored is suddenly released leading to branching cracks.

\subsection{Stress transmission}

The stress tensor at each node $i$ can be computed by summing all contributions of connected bonds $j$ using [54]

$\sigma_{i}=\frac{1}{2 V_{i}} \sum_{j \in \mathcal{H}_{i}} \boldsymbol{\xi}_{i j} \otimes \boldsymbol{F}_{i j}$

where $\boldsymbol{F}_{i j}$ is the force exerted on node $i$ by node $j$. Figure 5 shows the horizontal $\sigma_{x x}$ and vertical $\sigma_{y y}$ components of the stress fields. The maximum absolute values of these fields are located on the vertical symmetry axis of the particle. In compression, this maximum occurs in the vicinity of the contacts with the plates and in tension at the center of the particle.

Figure 6 shows peridynamics computed values, represented by circles together with two theoretical models. The horizontal and vertical stresses are normalized by the theoretical tensile stress at the center of the particle $\sigma_{t}=\frac{1}{\pi} \frac{F}{R}$ as a function of $y / R$ (for $x=0$ ). $\sigma_{y y}$ is always in compression (negative values) and is maximal at the contact with the plate and minimal at the center. The horizontal stress $\sigma_{x x}$ can be either negative or positive and reaches its maximum in traction close to the center. The first theoretical model assumes that the contact forces are applied on two points at the bottom and top of the particle [55]. Figure 6 shows this model where

$\sigma_{x x}=\sigma_{t}=\frac{1}{\pi} \frac{F}{R}$

and

$\sigma_{y y}=-\frac{1}{\pi} \frac{F}{R}\left(\frac{4}{1-(y / R)^{2}}-1\right)$

plotted as dotted lines for all points $(x=0, y)$ on the vertical axis. In the center of the particle, we note a rather good agreement with peridynamics while the discrepancy increases with distance to the center of the particle. The second model, introduced by Hondros [56], takes into account the extension of the contact zone (solid lines in Fig. 6). For each contact, the force is assumed to be uniformly distributed on an arc of size $2 \alpha$ on both sides of the contact. This model valid for small values of $\alpha$ leads to

$\sigma_{x x}=\frac{1}{\pi} \frac{F}{R}(u-v) / \alpha$ 

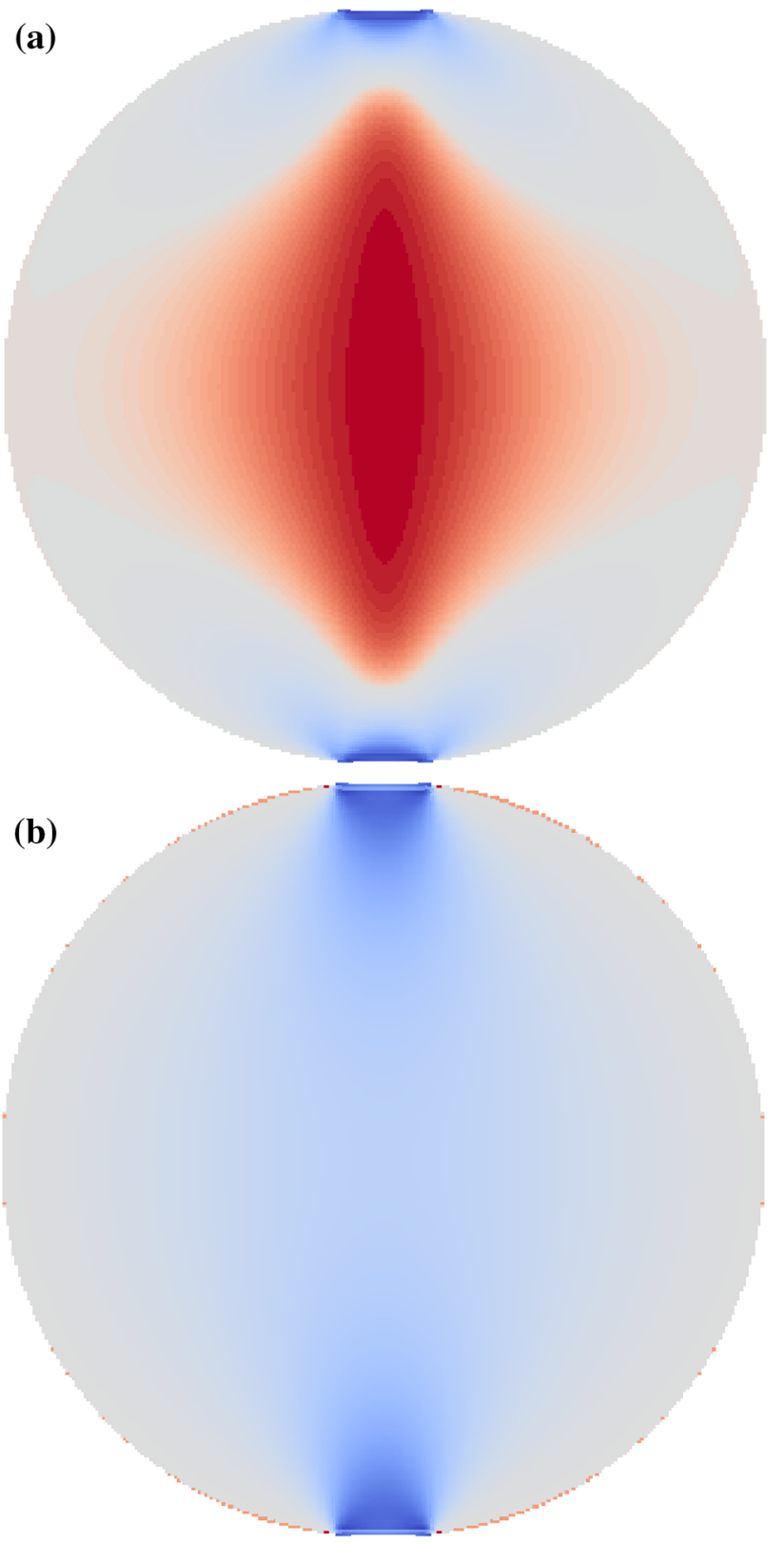

Fig. 5 Components $\mathbf{a} \sigma_{x x}$ and $\mathbf{b} \sigma_{y y}$ of the stress field for a particle submitted to diametral compression between two plates. The color gradient ranges from blue for maximal compression to red for maximal tensile stress and the mesh resolution is $N_{x}=512$

and

$\sigma_{y y}=-\frac{1}{\pi} \frac{F}{R}(u+v) / \alpha$

where

$u=\frac{1-(y / R)^{2}}{1-2(y / R)^{2} \cos 2 \alpha+(y / R)^{4}} \sin 2 \alpha$
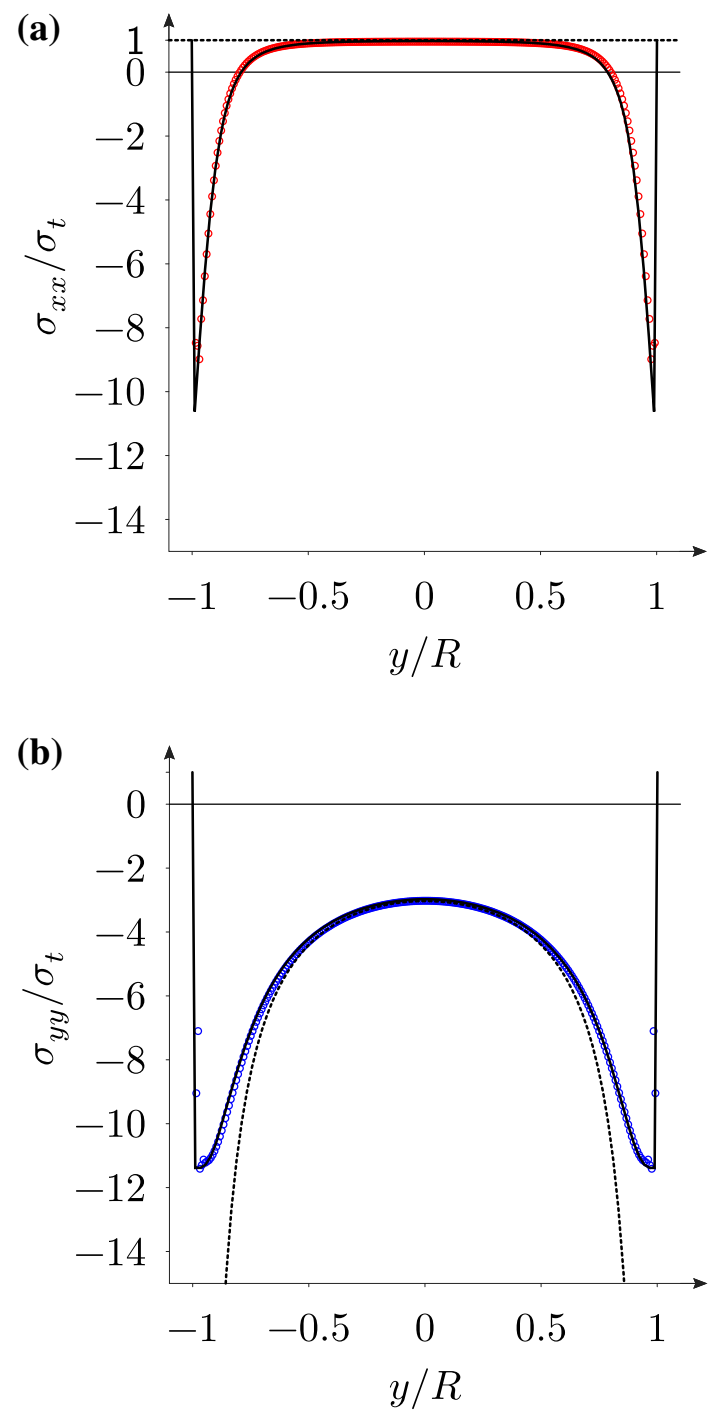

Fig. 6 Stress components $\sigma_{x x}$ (red circles) (a) and $\sigma_{y y}$ (blue circles) (b) normalized by $\sigma_{t}$ as a function vertical position along the vertical axis $y / R$. The solid lines and dotted lines correspond to distributed-loading model with an adjusted value of the angle $\alpha \simeq 8^{\circ}$ [see Eqs. (9), (10)] and point loading model (Eqs. 7, 8), respectively

and

$v=\tan ^{-1}\left(\frac{1+(y / R)^{2}}{1-(y / R)^{2}} \tan \alpha\right)$

We get a nice fit of the stress profiles $\sigma_{x x}$ and $\sigma_{y y}$ as shown in Fig. 6 by adjusting the value of $\alpha$. We also evaluated its value directly from the simulations for a particle with $N_{x}=256$ by considering the nodes in contact with the top plateau. The value that we get in this way is by $13 \%$ below the adjusted value. This can be explained by the fact that, despite the difference between the model and simulations in the way the wall force is applied, in both cases we have a diametral compression with free boundaries and strong stress concen- 
tration along the central axis. For this reason, the stress fields are similar up to a small adjustment of the model parameter $\alpha$.

\subsection{Discretization effects on Young's modulus}

In the literature, the sensitivity of macroscopic behavior to spatial discretization has been studied for various numerical methods and mesh conditions. For example, it has been shown that the elastic modulus converges toward a theoretical value when the spatial resolution tends to zero in the case of foam using Finite Elements Method [57], of the compaction of deformable particles using Material Point Method [58] or for pores and particle-embedded composites using Lattice Elements Method [3]. For peridynamics, this issue was addressed as a function of $h$ and $\delta x$ for homogeneous materials [41] and for a single-notched sample [59].

Eight diametral compression tests were performed for the same size of the particle $D=2 R$ with resolutions ranged from $N_{x}=256$ to $N_{x}=704$ nodes. In all simulations, the horizon was $h=3 \delta x$ and the stiffness $K$ was derived from the stress-strain curves $\sigma=K \varepsilon$ by considering the maximum slope before failure. Figure 7 shows $K$ normalized by the Young modulus $E$ as a function of $1 / N_{x}$. For elastic particles, the Hertz formula gives the relationship between the applied force $F$ and the elastic deflection $\delta$ at contact. For a $2 \mathrm{D}$ disk in contact with a rigid plate, $F=\frac{\pi}{4} E \delta$. As we consider, a grain compressed between two plates, $\delta=\Delta \ell / 2$ for each contact, where $\Delta \ell$ is the cumulated macroscopic displacement. Thus, dividing Hertz expression by $D$ we get $\sigma=K^{\text {th }} \varepsilon$ where $\frac{K^{\text {th }}}{E}=\frac{\pi}{8} \simeq 0.393$. Interestingly, in Fig. 7, when $1 / N_{X}$ tends to zero, $K / E$ can be linearly extrapolated to $\simeq 0.388$ independently of mesh size. This value which can be extrapolated with reasonable accuracy from a limited number of tests is slightly lower than $K^{\text {th }} / E$.

\section{Fracture of particles embedding defects}

In this section, we consider a particle in which 1D cracks are introduced by modifying the elastic properties of the bonds crossing a predefined flaw. The cracks act as inclusions with a lower stiffness than the original material. An interesting feature is the possibility to vary this stiffness from 0 to that of the matrix, where 0 corresponds to a perfect crack, and a value between 0 and the stiffness of the matrix to a partially detached crack. Figure 8 shows in gray level the density of bonds affected by this procedure. The ratio between the Young modulus of bonds crossing the defects and that of the matrix is set to 0.2 . We keep the same value of the critical elongation $s_{0}$ for all cracks independently of their positions although it has recently been suggested that its value may be

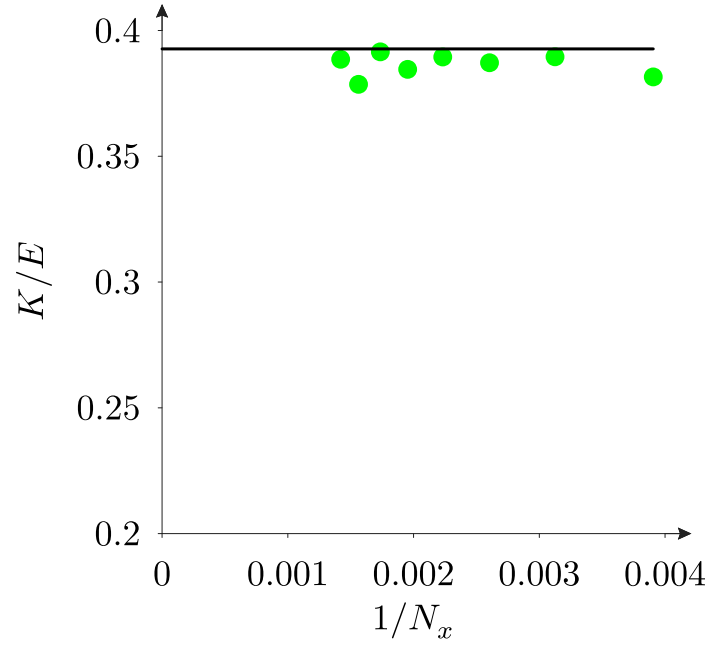

Fig. 7 Evolution of $K / E$ as a function of $1 / N_{x}$

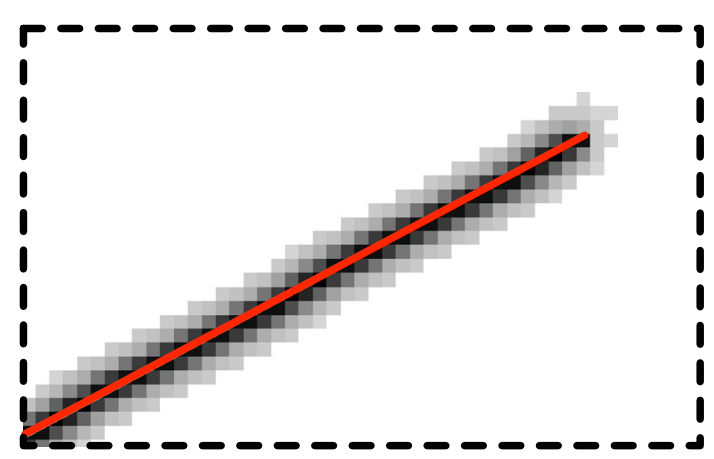

Fig. 8 Magnification of a defect in red. The material points are represented in gray levels, showing the total number of bonds per node which cross the defect. The scale ranges from white to black

different at the edges [60]. In a similar vein, as a first-order approximation, the micromodulus $c$ was kept constant over the whole domain. Corrections regarding surface effects on both elasticity and fracture properties may be found in [61]. In our case, the cracks are essentially initiated in the bulk where the flaws are distributed.

\subsection{Breakage of particles including a single vertical defect}

The presence of a defect and its position play an important role in the breakage of particles. Figure 9 shows an example of stress fields $\sigma_{x x}$ and $\sigma_{y y}$ in a particle with a vertical defect of length $0.1 D$ located at its center. We observe strong stress concentrations in compression in the vicinity of contacts and in tension at the tips of the flaw. We also see that the stresses spread over a large zone between the two tips.

In order to quantify the influence of the position of this defect on the yield stress of the particle, we performed a parametric study with more than 100 compression tests until rupture for a resolution $N_{x}=256$. The weakening effect of 

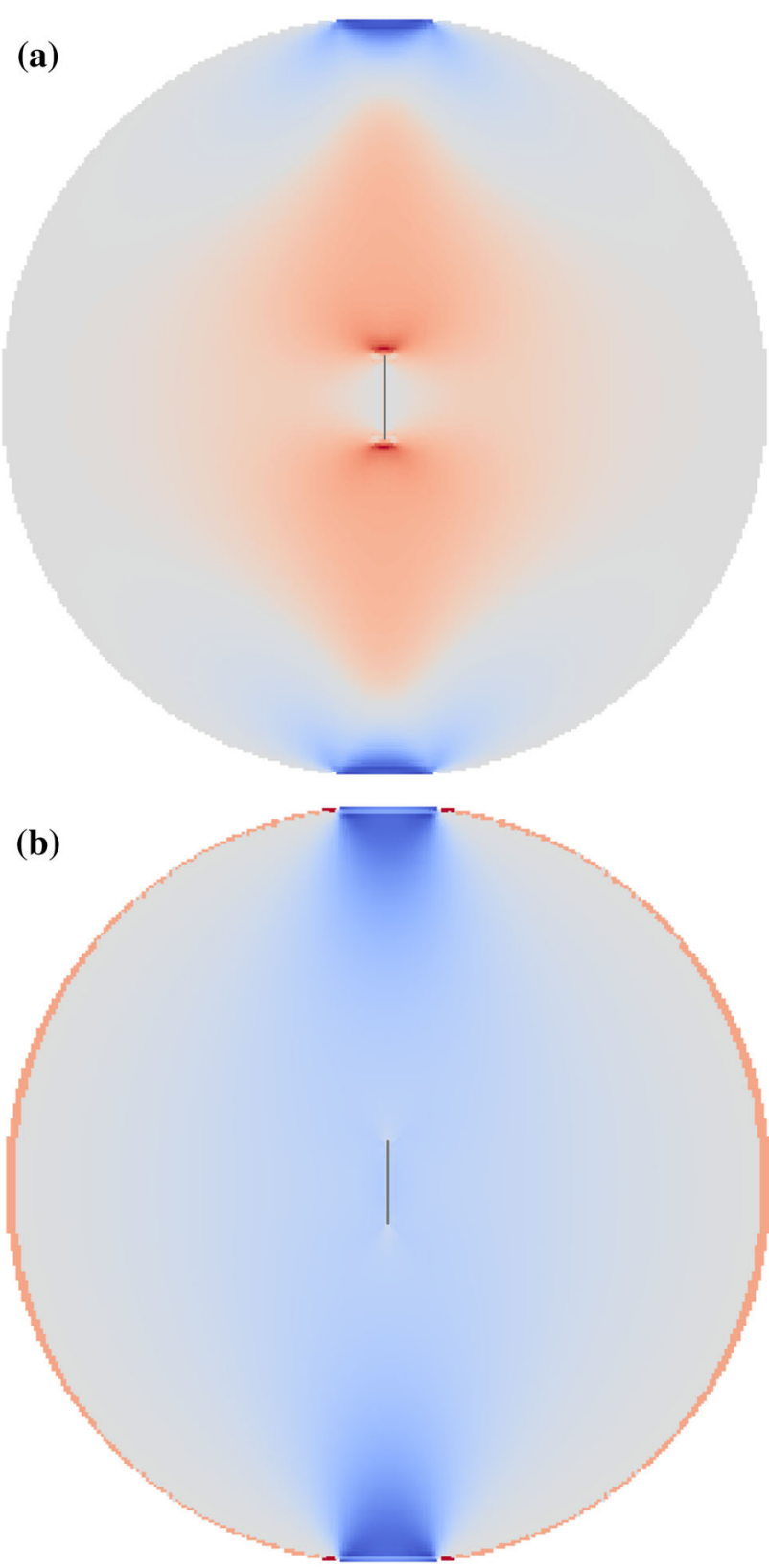

Fig. 9 Components $\mathbf{a} \sigma_{x x}$ and $\mathbf{b} \sigma_{y y}$ of the stress field for a particle under diametral compression embodying a single vertical defect at its center. The color scale ranges from blue (compression) to red (in tension)

defects on the particle is quantified using the parameter

$\zeta=\frac{\sigma^{Y}}{\sigma_{0}^{Y}}$

where $\sigma_{0}^{Y}$ is the failure stress for a material without defect (with the same meshing conditions). We have $\zeta \in[0,1]$ with $\zeta=1$ if the defect has no influence on the failure stress of the particle.

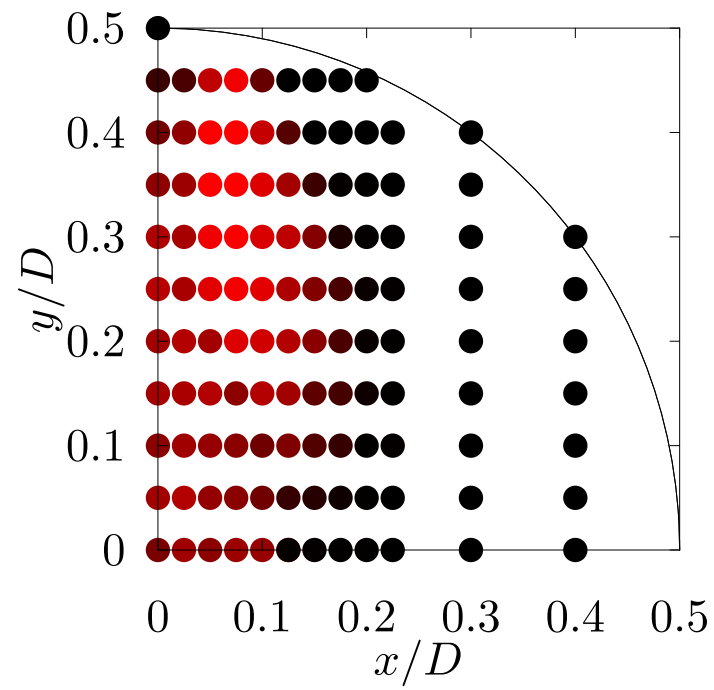

Fig. 10 Map of the influence of the position of a vertical defect on the yield stress of particles. The dots show the locations of the centers of the defects. The weakening effect of the defect is represented in red levels from black for no effect to red for maximum effect $(\zeta=0.55)$

Figure 10 shows a map of $\zeta$ values for various locations of defects in the particle. Due to axial symmetry, only a quarter of particle is considered. This map reveals two interesting features: (1) The critical zone is neither at the center of the particle nor on the vertical axis, and the maximal influence of the defect is around $(x / D=0.075, y / D=0.35)$; (2) For a distance on the $\mathrm{x}$-axis above $x / D \simeq 0.2$, the defect has a negligible impact on $\sigma^{Y}(\zeta=1)$. This means that the flaws and surface irregularities occurring at such distances from the vertical axis are not relevant to the fracture behavior of particles, whereas the same flaws along the axis are critical for the strength of the particle.

\subsection{Rupture of particles embedding a population of defects}

In this section, we consider particles of diameter $D=1$ embedding a population of defects of number density $\bar{n}=$ $100 \pi / 4$ with random orientation and length. The coordinates of the crack centers are randomly drawn in $[-D / 2, D / 2]$ following a multivariate uniform distribution. The length of defects follows a well-peaked normal distribution $d \sim$ $\mathcal{N}\left(\mu_{d} / D=0.035, \varsigma_{d} / D=0.005\right)$, where $\mu_{d}$ is the mean defect size and $\varsigma_{d}$ is its standard deviation. By well peaked, we mean a small ratio $\varsigma_{d} / \mu_{d} \simeq 0.14$. Figure 11a shows an example of these particles and Fig. 11b its horizontal stress component $\sigma_{x x}$ before failure for a particle subjected to compression between two plates. Compared to the horizontal stress field plotted in Fig. 5a, the population of defects generates fluctuations which may either concentrate or decrease the magnitude of the stress in the vicinity of defects. Note 


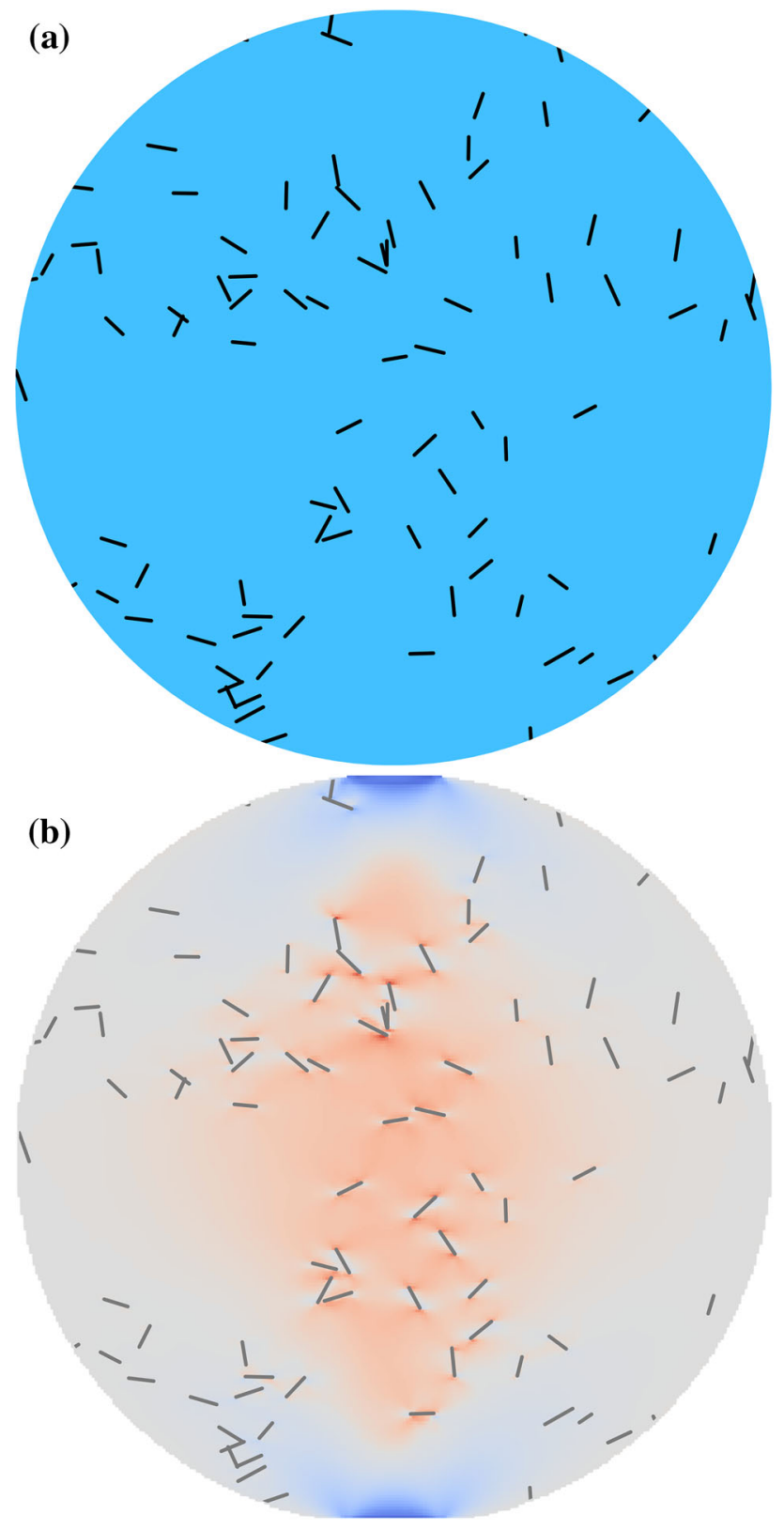

Fig. 11 Particle embedding a defect population. a Position of defects in the particle and $\mathbf{b}$ horizontal stress $\sigma_{x x}$

that crack propagation strongly depends on spatial stress resolution around the cracks, and therefore, the thickness and lengths of the cracks should be larger than the value of horizon used in the simulations. This is the case in our simulations and, as shown in Fig. 11b, the stress field is rather smooth even in the vicinity of the cracks.

When rupture occurs, these fluctuations are at the origin of an asymmetrical crack propagation (Fig. 12) which mainly split particles in two parts. We note also in this image that some cracks are at the origin of secondary fractures which do not fully propagate.

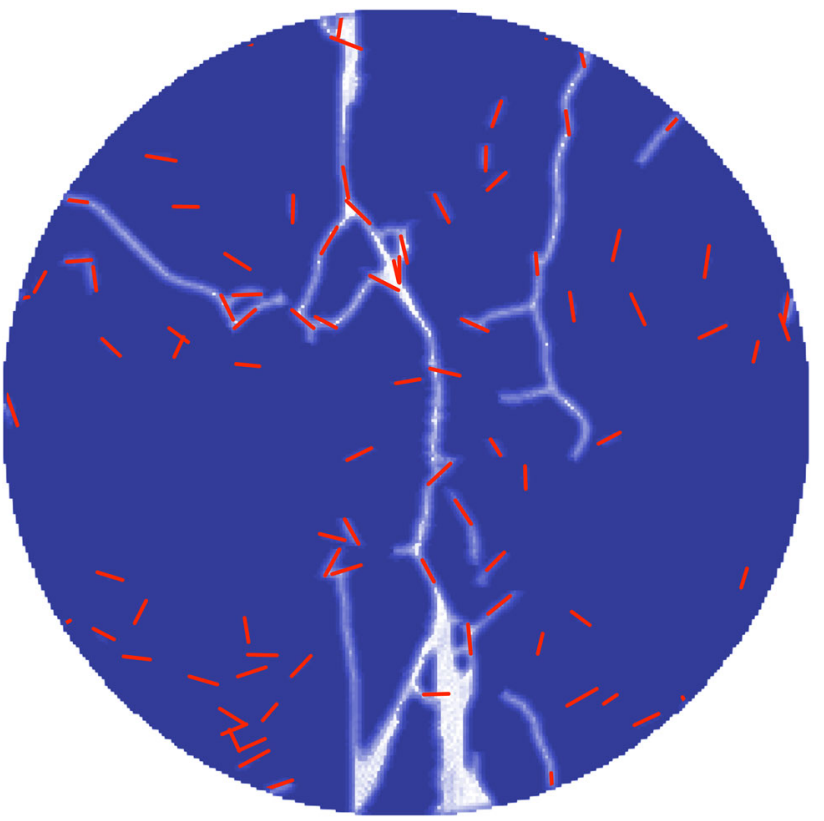

Fig. 12 Damage field after breakage (from blue for 0 damage to white for $100 \%)$. In red, the initial position of defects is shown

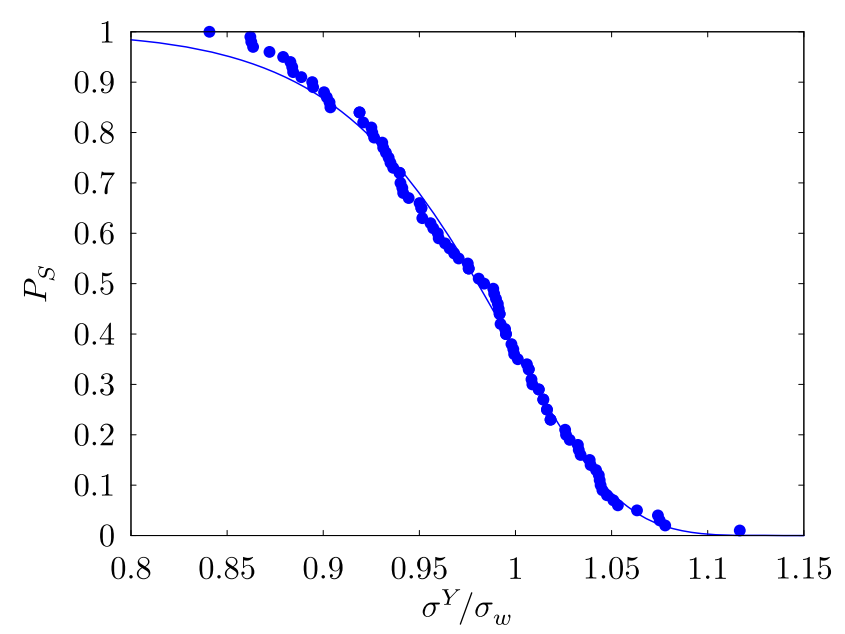

Fig. 13 Survival probability of particles as a function of the applied stress. The solid line represents Weibull fits to the data

In order to study the dispersion of the values of the yield stress, we consider the probability for a particle to survive to a specific loading stress. For $N$ particles, the probability $P_{\mathrm{S}}(i)=1-\frac{i}{N}$ is assigned to the $i$ th smallest yield stress value. Figure 13 shows this distribution and a fit by the Weibull law

$P_{\mathrm{S}}=\mathrm{e}^{-\left(\sigma^{Y} / \sigma_{\mathrm{w}}\right)^{m}}$

In this expression, $\sigma_{\mathrm{w}}$ corresponds to the stress for which $63 \%$ of particles are broken and $m$ is the Weibull modulus characterizing the width of the distribution. Using Least Square 

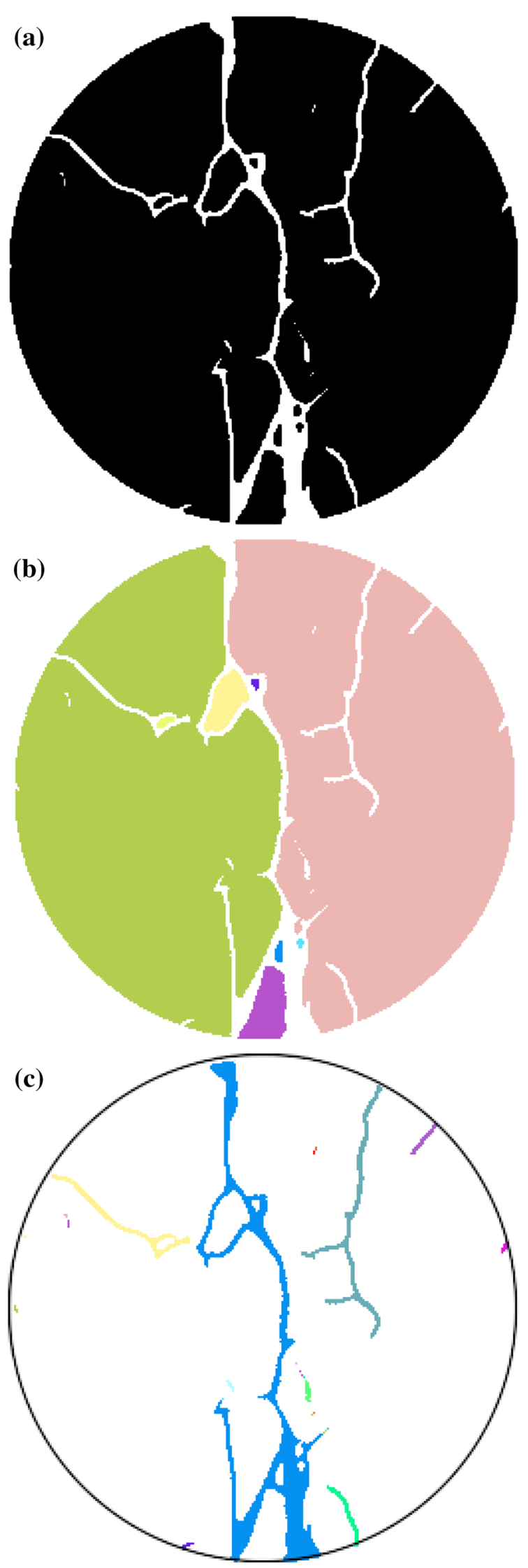

Fig. 14 a Binarized thresholded image based on the damage level (Fig. 12). Floodfill identification (colors indicates independent regions) of the fragments (b) and of cracks (c)

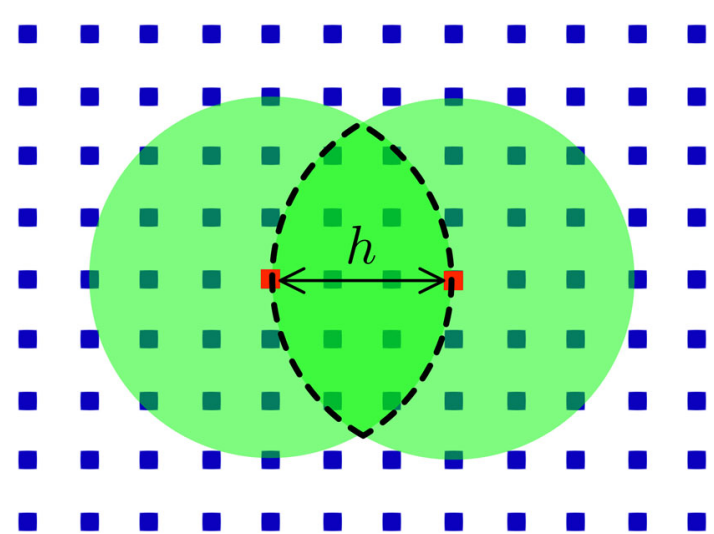

Fig. 15 Two nodes at a distance equal to the horizon $h$ and their respective horizons. The nodes linked to the two nodes belong to the intersection area

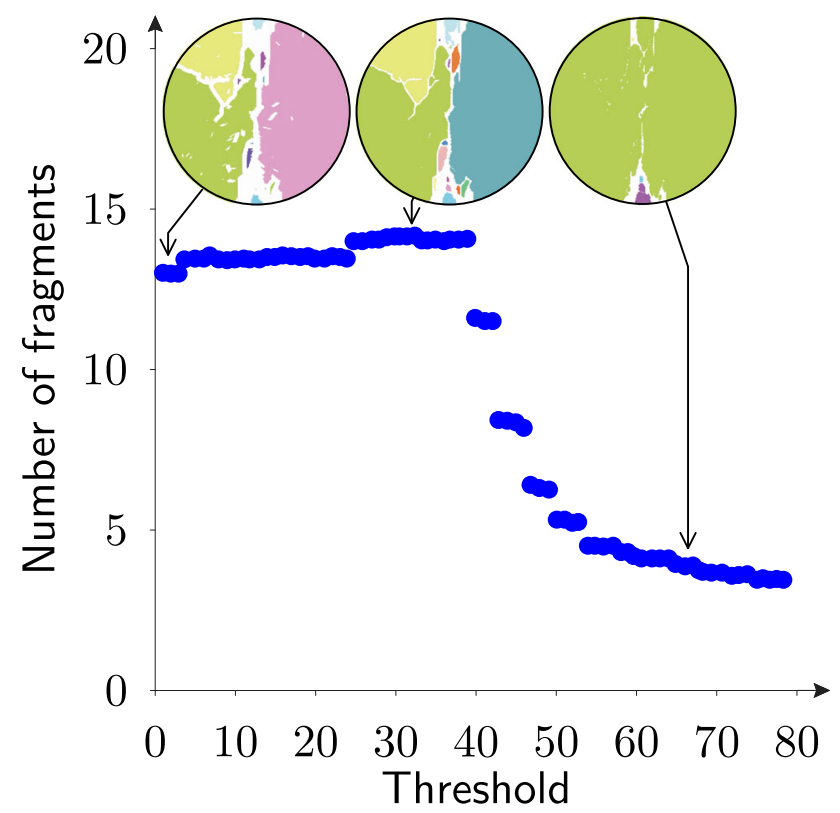

Fig. 16 Number of fragments as a function of damage threshold. Three examples of the fragment maps are displayed for three different values of damage threshold

Method, $m$ was found to be equal to 18.5 which is in the range of ceramics materials [62].

\subsection{Statistical analysis of fragments and cracks}

A "floodfill" algorithm [63,64] is used to create maps of fragments where each fragment is labeled by a unique color number (Fig. 14b). From a computational point of view, this color map is a $2 \mathrm{D}$ table map $[\mathrm{x}][\mathrm{y}]$ initialized with a binarized image (Fig. 14a) obtained from the damage levels (Fig. 12). 
Fig. 17 Colored fragments of all tested particles. Colors are chosen randomly

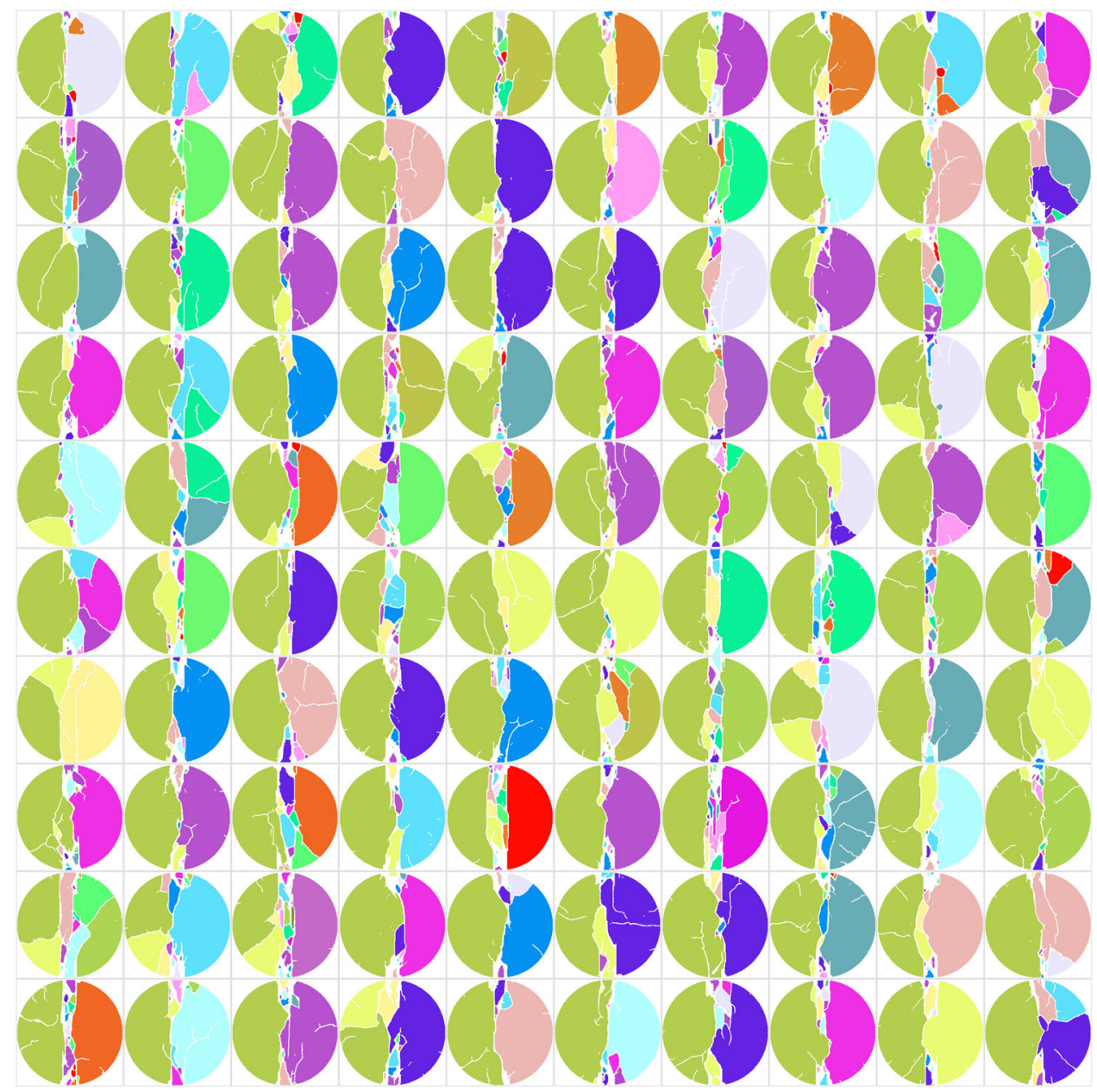

In our simulations, we attributed the black color for all material points with a damage below a threshold of 30\% corresponding to fragments and white otherwise, corresponding to crack pattern. This choice is based on the fact that the interactions between nodes and thus the threshold for breakage should be consistent with the length scale of mechanical interactions, which in Peridynamics is governed by the horizon $h$. Two nodes separated by a distance $h$ interact through a direct link but also through all others nodes that are linked to the two nodes. As illustrated in Fig. 15, these mediating nodes belong to the intersection area of the horizons of the two nodes, and thus, they represent a fraction

$P_{d}=\frac{2}{3}-\frac{\sqrt{3}}{2 \pi} \simeq 0.39$

of the total area of all bonds inside the horizon of a bond. This implies that for two nodes separated at a distance $h$, at least $39 \%$ of bonds should be removed in order to avoid the transmission of information between the two nodes through a neighboring node. In practice, we used the slightly lower value of $30 \%$ for the analysis of the fragments. With this choice, the sizes of the fragments are expected to be larger than $h$. The crack patterns are practically similar for the fractions of $30 \%$ and $39 \%$. Figure 16 shows an example of the number of fragments as a function of the threshold. We see that, as because of an increasingly finer resolution, the number of fragments slightly increases as the threshold is increased from zero, but beyond 39\% the number of fragments declines rapidly with threshold since there are less cracks.

In the floodfill algorithm, the connected nodes are identified as fragments. Hence, each fragment is surrounded by cracks, and two different fragments have no common node. The method consists in flooding the connected black regions with a new color "color" according to the following routine:

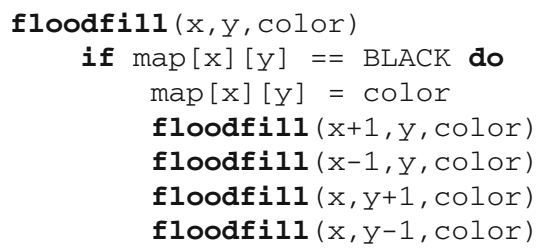



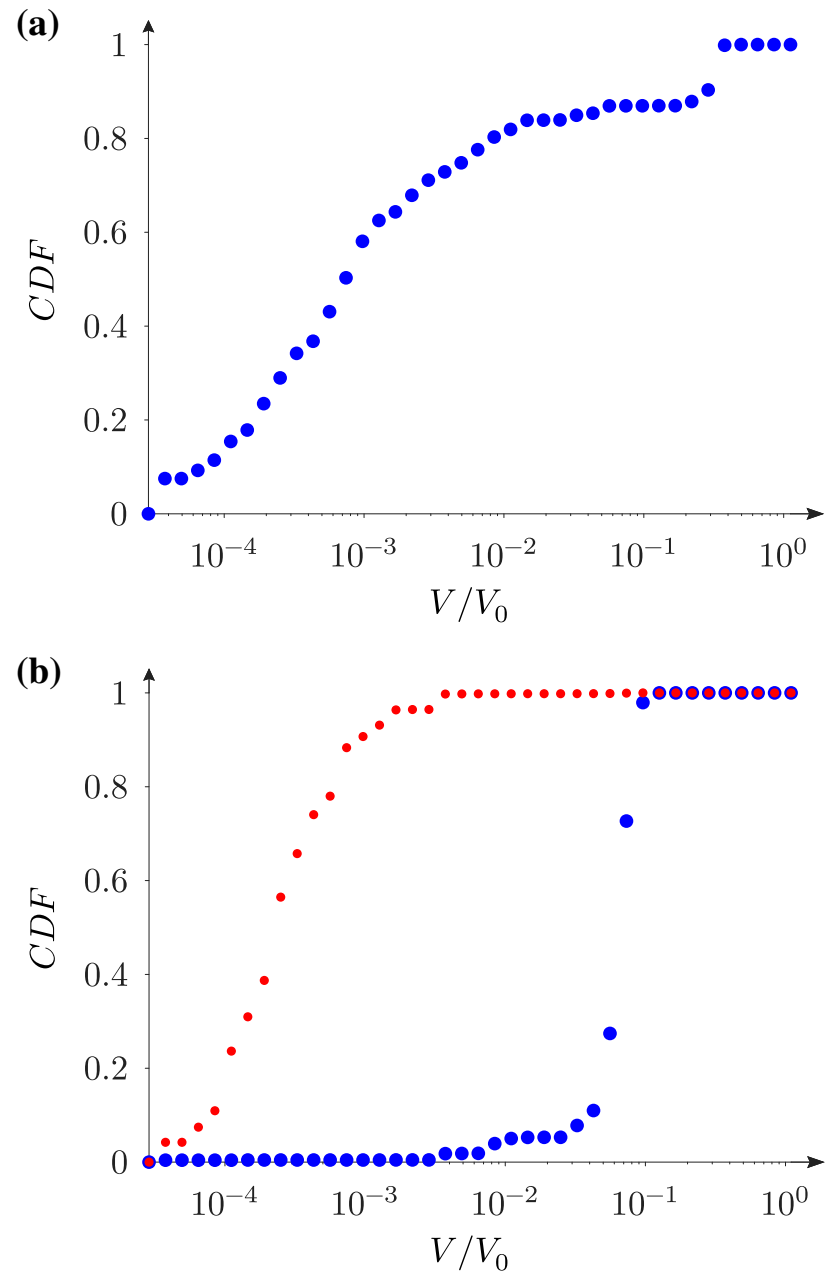

Fig. 18 a Cumulative distribution function of fragment sizes. b Cumulative distribution function of crack sizes in red and of the cracks at the origin of fragments in blue. (Color figure online)

end if

end floodfill

In this procedure, all $(\mathrm{x}, \mathrm{y})$ are called with a new color id color. Note that the order the pixels are called does not change the result. The same procedure is used to identify the cracks (Fig. 14c). Figure 17 shows the fragment identification performed on 100 flawed particles the same statistical distribution of the pre-cracks.

The cumulative distribution function (CDF) of the volumes $V$ of the fragments, normalized by the initial volume of the particle $V_{0}$, is plotted in Fig. 18a. We see two different populations of fragments: a broad distribution of smallest pieces, representing nearly $7 \%$ of the initial particle volume and a narrowly distributed zone centered on $40 \%$. This distribution includes fragments of all sizes and a few very large fragments.
Figure 18b (blue curve) displays the CDF of crack volumes. We distinguish two types of cracks: (a) cracks that are located inside a fragment and (b) cracks that are at the interface between fragments. The latter are the cracks that created the fragments by their propagation. We see that the fragments are mostly related to the main crack splitting the particle into two parts. In most cases, the smallest fragments are located in this central zone as observed in Fig. 17. The cracks of the first kind can be found also near the central crack, but also far from it. They represent the cracks that did not propagate long enough to create fragments. They are often quite small with an average volume of $0.03 \%$ of the particle volume. We clearly see the difference between the cumulative volumes of the two kinds of cracks in Fig. 18b.

These results show that, despite the presence of defects, a particle breaks mainly due to the propagation of a crack along the central axis, as expected from a classical analysis. However, we also see that defects and disorder lead to diffuse crack patterns that can create many more fragments of different sizes.

\section{Conclusions}

In this paper, we used a 2D bond-based peridynamics method to investigate the breakage of particles containing defects. In the case of particles without defects, we showed that, even if the rectilinear meshing of the particle may lead to a poor description of the curvature at the contact scale, the horizontal and vertical stress fields are well predicted. Furthermore, for an increasingly resolved meshing, the particle stiffness converges linearly to a well-defined value close to the theoretical value obtained using Hertz equation for the contact between a disk and a plate in plan strain. A model of defect was introduced, based on a weakening of the elastic modulus of elements crossing a 1D linear notch. More than 100 compression tests were performed to investigate the influence of the location of a vertical defect in the upper right quadrant of a disk-particle. Interestingly, we show that the lowest yield stress does not occur for a position of the defect at the center of the particle.

We also characterized the evolution of yield stress for 100 particles with the same statistical population of defects and different random draws. We found that the yield stress follows a Weibull distribution whose exponent $m \simeq 18$ is consistent with that of ceramic's materials. A floodfill procedure was then employed to determine the volumes occupied by fragment and cracks and aggregated data allowed us to plot the corresponding probability distributions for the whole set of particles.

In future work, we would like to analyze more deeply the link between the parameters controlling the population of defects at local scale and the global probability of survivals 
of particles. A key feature of the peridynamics method is its ability to simulate highly dynamical problems as breakage under impact. Another perspective is the effect of the deformation rate on the fragmentation of particles.

Acknowledgements We are grateful to the INRAE MIGALE bioinformatics facility (MIGALE, INRAE, 2020. Migale bioinformatics Facility, doi: 10.15454/1.5572390655343293E12) and to the genotoul bioinformatics platform Toulouse Occitanie (Bioinfo Genotoul, doi: $10.15454 / 1.5572369328961167 \mathrm{E} 12$ ) for providing computing and storage resources.

\section{Compliance with ethical standards}

Conflict of interest The authors report no conflict of interest in this study.

\section{References}

1. Song B, Rough S, Wilson D (2007) Effects of drying technique on extrusion-spheronisation granules and tablet properties. Int $\mathbf{J}$ Pharm 332(1-2):38

2. Fichtner F, Rasmuson $\AA$, Alderborn G (2005) Particle size distribution and evolution in tablet structure during and after compaction. Int J Pharm 292(1-2):211

3. Affes R, Delenne JY, Monerie Y, Radjai F, Topin V (2012) Tensile strength and fracture of cemented granular aggregates. Eur Phys $\mathbf{J}$ E 35(11): 117

4. Torquato S (2002) Random heterogeneous materials. Springer, Berlin

5. Aliha M, Ayatollahi M (2014) Rock fracture toughness study using cracked chevron notched Brazilian disc specimen under pure modes I and II loading-A statistical approach. Theor Appl Fract Mech 69:17

6. Guo H, Aziz N, Schmidt L (1993) Rock fracture-toughness determination by the Brazilian test. Eng Geol 33(3):177

7. Zhou XP, Wang YT (2016) Numerical simulation of crack propagation and coalescence in pre-cracked rock-like Brazilian disks using the non-ordinary state-based peridynamics. Int J Rock Mech Min Sci 89:235

8. Khanal M, Schubert W, Tomas J (2005) Experiment and simulation of breakage of particle compounds under compressive loading. Part Sci Technol 23(4):387

9. Xiao Y, Sun Z, Desai CS, Meng M (2019) Strength and surviving probability in grain crushing under acidic erosion and compression. Int J Geomech 19(11):04019123

10. Dan DQ, Konietzky H, Herbst M (2013) Brazilian tensile strength tests on some anisotropic rocks. Int J Rock Mech Min Sci 58:1

11. Tavallali A, Vervoort A (2013) Behaviour of layered sandstone under Brazilian test conditions: layer orientation and shape effects. J Rock Mech Geotech Eng 5:366

12. Zubelewicz A, Bažant ZP (1987) Interface element modeling of fracture in aggregate composites. J Eng Mech 113(11):1619

13. Kun F, Herrmann HJ (1996) A study of fragmentation processes using a discrete element method. Comput Methods Appl Mech Eng 138(1-4):3

14. Åström JA, Herrmann HJ (1998) Fragmentation of grains in a twodimensional packing. Eur Phys J B Condens Matter Complex Syst 5(3):551
15. Tsoungui O, Vallet D, Charmet JC (1999) Numerical model of crushing of grains inside two-dimensional granular materials. Powder Technol 105(1-3):190

16. Nguyen DH, Sornay P, Azéma E, Radjai F (2015) Evolution of particle size distributions in crushable granular materials. Geomech Micro Macro 1:275

17. Nguyen DH, Azéma E, Sornay P, Radjai F (2015) Bonded-cell model for particle fracture. Phys Rev E 91(2):022203

18. Orozco LF, Nguyen DH, Delenne JY, Sornay P, Radjai F (2019) Discrete-element simulations of comminution in rotating drums: effects of grinding media. Powder Technol 362:157-167

19. Ciantia M, de Toledo M, Alvarez Arroyo, Calvetti F, Gens Solé A (2015) An approach to enhance efficiency of DEM modelling of soils with crushable grains. Géotechnique 65(2):91

20. Zhou W, Yang L, Ma G, Chang X, Cheng Y, Li D (2015) Macromicro responses of crushable granular materials in simulated true triaxial tests. Granul Matter 17(4):497

21. Galindo-Torres S, Pedroso D, Williams D, Li L (2012) Breaking processes in three-dimensional bonded granular materials with general shapes. Comput Phys Commun 183(2):266

22. Orozco LF, Delenne JY, Sornay P, Radjai F (2019) Discreteelement model for dynamic fracture of a single particle. Int J Solids Struct 166:47

23. Radi K, Jauffrès D, Deville S, Martin CL (2019) Elasticity and fracture of brick and mortar materials using discrete element simulations. J Mech Phys Solids 126:101

24. Kertész J (1990) 8 - Dielectric breakdown and single crack models. In: Herrmann HJ, Roux S (eds) Statistical models for the fracture of disordered media. Random materials and processes. North-Holland, Amsterdam, pp 261-290

25. Topin V, Delenne JY, Radja1 F, Brendel L, Mabille F (2007) Strength and failure of cemented granular matter. Eur Phys J E 23(4):413

26. Nikravesh S, Gerstle W (2018) Improved state-based peridynamic lattice model including elasticity, plasticity and damage. Comput Model Eng Sci 116(3):323

27. Gerstle W, Sau N, Silling S (2007) Peridynamic modeling of concrete structures. Nucl Eng Des 237(12-13):1250

28. Celik E, Guven I, Madenci E (2011) Simulations of nanowire bend tests for extracting mechanical properties. Theor Appl Fract Mech 55(3): 185

29. Perré P, Almeida G, Ayouz M, Frank X (2016) New modelling approaches to predict wood properties from its cellular structure: image-based representation and meshless methods. Ann For Sci 73(1): 147

30. Silling SA (2000) Reformulation of elasticity theory for discontinuities and long-range forces. J Mech Phys Solids 48(1):175

31. Ha YD, Bobaru F (2011) Characteristics of dynamic brittle fracture captured with peridynamics. Eng Fract Mech 78(6):1156

32. Love AEH (1944) A treatise on the mathematical theory of elasticity. Dover Publications, Mineola

33. Herrmann HJ, Roux S (1990) Statistical models for the fracture of disordered media. Elsevier, Amsterdam

34. Laubie H, Monfared S, Radjaï F, Pellenq R, Ulm FJ (2017) Effective potentials and elastic properties in the lattice-element method: isotropy and transverse isotropy. J Nanomech Micromech 7(3):04017007

35. Sarego G, Le QV, Bobaru F, Zaccariotto M, Galvanetto U (2016) Linearized state-based peridynamics for 2-D problems. Int J Numer Methods Eng 108(10):1174

36. Zhu Q, Ni T (2017) Peridynamic formulations enriched with bond rotation effects. Int J Eng Sci 121:118

37. Brandrup J, Immergut EH, Grulke EA, Abe A, Bloch DR (1999) Polymer handbook, vol 89. Wiley, New York

38. Comte C, von Stebut J (2002) Microprobe-type measurement of Young's modulus and Poisson coefficient by means of depth 
sensing indentation and acoustic microscopy. Surf Coat Technol 154(1):42

39. Ridha S, Hamid AIA, Halim AHA, Zamzuri NA (2018) Elasticity and expansion test performance of geopolymer as oil well cement. IOP Conf Ser Earth Environ Sci 140:012147. https://doi.org/10. 1088/1755-1315/140/1/012147

40. Allen M, Tildesley D (1986) Computer simulation of liquids. Oxford University Press, Oxford

41. Freimanis A, Paeglitis A (2017) Mesh sensitivity in peridynamic quasi-static simulations. Procedia Eng 172:284

42. Yaghoobi A (2015) Meshless modeling framework for fiber reinforced concrete structures. Comput Struct 161:43

43. Indriyantho BR (2014) Finite element modeling of concrete fracture in tension with the Brazilian splitting test on the case of plane-stress and plane-strain. Procedia Eng 95:252

44. Vo TT, Mutabaruka P, Nezamabadi S, Delenne JY, Izard E, Pellenq R, Radjai F (2018) Mechanical strength of wet particle agglomerates. Mech Res Commun 92:1

45. Wu S, Ma J, Cheng Y, Xu M, Huang X (2018) Numerical analysis of the flattened Brazilian test: failure process, recommended geometric parameters and loading conditions. Eng Fract Mech 204:288

46. Nguyen DH, Azéma E, Sornay P, Radjai F (2015) Bonded-cell model for particle fracture. Phys Rev E 91:022203

47. Wu L, Huang D, Xu Y, Wang L (2019) A non-ordinary statebased peridynamic formulation for failure of concrete subjected to impacting loads. Comput Model Eng Sci 118(3):561

48. Wang Y, Han F, Lubineau G (2019) A hybrid local/nonlocal continuum mechanics modeling and simulation of fracture in brittle materials. Comput Model Eng Sci 121:399-423

49. Raymond SJ, Jones BD, Williams JR (2019) Modeling damage and plasticity in aggregates with the material point method (MPM). Comput Part Mech 6(3):371

50. Bilgen C, Homberger S, Weinberg K (2019) Phase-field fracture simulations of the Brazilian splitting test. Int J Fract 220(1):85

51. Luding S (2008) Cohesive, frictional powders: contact models for tension. Granul Matter 10(4):235

52. Timoshenko S, Goodier J (1969) Theory of elasticity. McGrawHill, New York
53. Lin H, Xiong W, Zhong W, Xia C (2014) Location of the crack initiation points in the Brazilian disc test. Geotech Geol Eng 32(5):1339

54. Laubie H, Monfared S, Radjai F, Pellenq R, Ulm FJ (2017) Disorder-induced stiffness degradation of highly disordered porous materials. J Mech Phys Solids 106:207

55. Jianhong Y, Wu F, Sun J (2009) Estimation of the tensile elastic modulus using Brazilian disc by applying diametrically opposed concentrated loads. Int J Rock Mech Min Sci 46(3):568

56. Hondros G (1959) The evaluation of Poisson's ratio and the modulus of materials of a low tensile resistance by the Brazilian (indirect tensile) test with particular reference to concrete. Aust J Appl Sci 10(3): 243

57. Roberts AP, Garboczi EJ (2016) Elastic properties of model random three-dimensional open- cell solid. J Mech Phys Solids 50:33

58. Nezamabadi S, Radjai F, Averseng J, Delenne JY (2015) Implicit frictional-contact model for soft particle systems. J Mech Phys Solids 83:72

59. Frank X, Radjaï F, Nezamabadi S, Delenne JY (2020) Tensile strength of granular aggregates: stress chains across particle phase versus stress concentration by pores. Phys Rev E 102:022906

60. Yu H, Li S (2020) On energy release rates in peridynamics. J Mech Phys Solids 142:104024

61. Le QV, Bobaru F (2018) Surface corrections for peridynamic models in elasticity and fracture. Comput Mech 61(4):499

62. Stoyan D, Gloaguen R (2011) Nucleation and growth of geological faults. Nonlinear Process Geophys 18(4):529

63. Haig TD, Attikiouzel Y, Alder M (1992) Border following new definition gives improved borders. IEE Proc I (Commun Speech Vis) 139(2):206

64. Delenne JY, Richefeu V, Radjai F (2015) Liquid clustering and capillary pressure in granular media. J Fluid Mech 762:R5 